\title{
Alterstice
}

Revue internationale de la recherche interculturelle

International Journal of Intercultural Research

Revista International de la Investigacion Intercultural

\section{Santé mentale et sociétés plurielles}

\section{Betty Goguikian Ratcliff et Ilario Rossi}

Volume 4, numéro 2, 2014

Santé mentale et sociétés plurielles

URI : https://id.erudit.org/iderudit/1077421ar

DOI : https://doi.org/10.7202/1077421ar

Aller au sommaire du numéro

\section{Éditeur(s)}

Alterstice

ISSN

1923-919X (numérique)

Découvrir la revue

Citer ce document

Goguikian Ratcliff, B. \& Rossi, I. (2014). Santé mentale et sociétés plurielles. Alterstice, 4(2), 3-12. https://doi.org/10.7202/1077421ar

\section{Résumé de l'article}

L'émergence de sociétés plurielles s'inscrit dans une mouvance historique et culturelle irréversible. Elle s'exprime notamment par la mobilité croissante des personnes, des savoirs, des pratiques et des techniques. Qu'elle soit politique, sociale, économique, culturelle, religieuse ou sexuelle, la diversité apparaît dès lors comme une exigence avec laquelle les équilibres de la démocratie, les enjeux de la société civile, les politiques publiques et les pratiques professionnelles doivent désormais composer. Cette situation engendre de nouvelles problématiques en matière de santé, et lance de nouveaux défis, notamment dans le domaine de la santé mentale. Ainsi, à une vision relativiste se limitant au respect des différences culturelles, se substitue progressivement un paradigme nouveau prônant une approche interculturelle, ou transculturelle. Celle-ci est davantage axée sur les trajectoires de vie, les processus dynamiques, les interactions et la mise en place de dispositifs cliniques allant dans le sens de l'ouverture de l'espace thérapeutique aux aspects de confrontation, de médiation et de métissage. Dans cette optique, la diversité, ainsi que les notions de culture, d'identité et d'hybridation qui s'y rattachent, sont incarnées et élaborées au sein même de la rencontre clinique, d'où émergent de nouveaux positionnements. Il s'agit de dépasser la centration sur la différence culturelle, notion qui, bien souvent, essentialise les différences et génère un sentiment de fossé infranchissable entre personnes de cultures différentes. À l'inverse, saisir les mutations en cours et reconnaître la diversité qui caractérise nos sociétés contemporaines permet de prendre en compte, dans le champ d'observation et d'analyse, l'éventail de nos propres acteurs et dispositifs. Sont ainsi esquissées quelques pistes de réflexion et d'analyse pour élucider les différents registres qui construisent et conditionnent la compréhension et l'utilisation du concept de diversité dans le domaine de la santé mentale. Il s'agit de cerner moins la relation entre santé et migration que le contexte au sein duquel les chercheurs et praticiens du champ de la santé mentale appréhendent la diversité et renouvellent leur propre culture professionnelle.
Ce document est protégé par la loi sur le droit d'auteur. L'utilisation des services d’Érudit (y compris la reproduction) est assujettie à sa politique d'utilisation que vous pouvez consulter en ligne.

https://apropos.erudit.org/fr/usagers/politique-dutilisation/ 


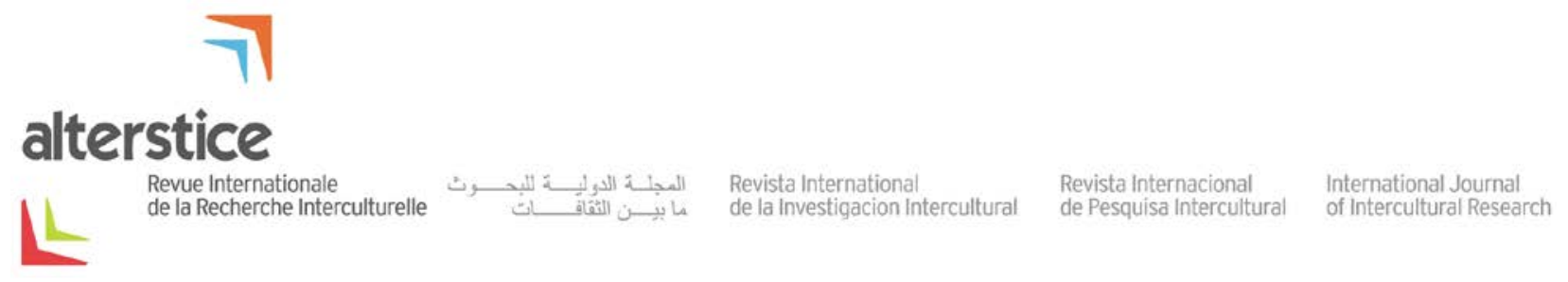

INTRODUCTION THÉMATIQUE

\section{Santé mentale et sociétés plurielles}

Betty Goguikian Ratcliff ${ }^{1}$ et Ilario Rossi ${ }^{2}$

\section{Résumé}

L'émergence de sociétés plurielles s'inscrit dans une mouvance historique et culturelle irréversible. Elle s'exprime notamment par la mobilité croissante des personnes, des savoirs, des pratiques et des techniques. Qu'elle soit politique, sociale, économique, culturelle, religieuse ou sexuelle, la diversité apparaît dès lors comme une exigence avec laquelle les équilibres de la démocratie, les enjeux de la société civile, les politiques publiques et les pratiques professionnelles doivent désormais composer. Cette situation engendre de nouvelles problématiques en matière de santé, et lance de nouveaux défis, notamment dans le domaine de la santé mentale. Ainsi, à une vision relativiste se limitant au respect des différences culturelles, se substitue progressivement un paradigme nouveau prônant une approche interculturelle, ou transculturelle. Celle-ci est davantage axée sur les trajectoires de vie, les processus dynamiques, les interactions et la mise en place de dispositifs cliniques allant dans le sens de l'ouverture de l'espace thérapeutique aux aspects de confrontation, de médiation et de métissage. Dans cette optique, la diversité, ainsi que les notions de culture, d'identité et d'hybridation qui s'y rattachent, sont incarnées et élaborées au sein même de la rencontre clinique, d’où émergent de nouveaux positionnements. II s'agit de dépasser la centration sur la différence culturelle, notion qui, bien souvent, essentialise les différences et génère un sentiment de fossé infranchissable entre personnes de cultures différentes. À l'inverse, saisir les mutations en cours et reconnaître la diversité qui caractérise nos sociétés contemporaines permet de prendre en compte, dans le champ d'observation et d'analyse, l'éventail de nos propres acteurs et dispositifs. Sont ainsi esquissées quelques pistes de réflexion et d'analyse pour élucider les différents registres qui construisent et conditionnent la compréhension et l'utilisation du concept de diversité dans le domaine de la santé mentale. II s'agit de cerner moins la relation entre santé et migration que le contexte au sein duquel les chercheurs et praticiens du champ de la santé mentale appréhendent la diversité et renouvellent leur propre culture professionnelle.

\section{Rattachement des auteurs}

${ }^{1}$ Faculté de psychologie et des sciences de l'éducation, Université de Genève, Suisse; ${ }^{2}$ Institut des sciences sociales, Université de Lausanne, Suisse

\section{Correspondance}

betty.goguikian@unige.ch et ilario.rossi@unil.ch

\section{Mots clés}

migration; santé mentale; sociétés plurielles; différence culturelle; métissage; diversité; relation de soin

\section{Pour citer cet article}

Goguikian Ratcliff, B. et Rossi, I. (2014). Santé mentale et sociétés plurielles. Alterstice, 4(2), 3-12. 
Ce numéro d'Alterstice convie des auteurs de disciplines différentes (médecine, psychiatrie, psychologie, sociologie, anthropologie, travail social) à avoir une réflexion commune à l'interface de deux notions d'actualité, santé mentale et sociétés plurielles. ${ }^{1}$. Bien sûr, l'une et l'autre de ces notions renvoient à des référentiels sémantiques distincts, et elles paraissent de prime abord fort éloignées, ne recoupant ni les mêmes niveaux d'analyse, ni les mêmes implications politiques et sociales. Mais, force est de constater qu'elles participent du même espace d'interdépendances et d'interconnexions, constitué de lieux, d'outils et de forces de mobilisation transnationales, dans lequel toute société pense et agit à des échelles plurielles (Abélès, 2008). Leurs relations se modifient en conséquence, et ce sont justement ces relations et leurs multiples angles de vue qui constituent le thème central de cette réflexion. Les raisons d'une telle posture sont multiples. Nous en avancerons ici deux, dont la valeur heuristique est transversale aux différentes perspectives et disciplines.

La première tient compte du contexte mondial, qui tend à se modifier avec rapidité. II n'est pas besoin de rappeler que I'on a désormais affaire à ce que l'on pourrait qualifier de nouvelle réalité planétaire (Hugo, 2005). La mondialisation des flux migratoires prend des proportions jamais atteintes auparavant, impliquant un nombre de plus en plus considérable de personnes, de familles et de diasporas en déplacement, que ce soit pour permettre des recompositions familiales, pour répondre à des demandes du marché du travail, ou encore en vue d'améliorer son sort, de trouver un lieu où s'établir et de vivre des socialisations renouvelées. Cette nouvelle donne mondiale en est à ses débuts et, avec ses problèmes et ses ressources, ses contradictions et ses opportunités, ce nouveau monde constitue autant un imaginaire qu'une réalité tangible (Laïdi, 2004). Certes, il n'a jamais existé de société figée dans l'Histoire, mais l'émergence des sociétés plurielles, notamment en Occident, s'exprime par une mobilité généralisée des personnes, des savoirs, des pratiques et des techniques, d'ici et d'ailleurs, ici et ailleurs. Ces assemblages créent des relations, des dépendances et des opportunités entre les individus, les communautés, les sociétés, les Étas et les continents (Collier et Ong, 2005). Ce qui paraît nouveau de nos jours, c'est le renforcement de cette mobilité à mesure que se poursuivent les transformations liées à la mondialisation : entre l'effacement progressif des frontières et la condition diasporique, une adéquation nouvelle se fait jour, celle des sociétés plurielles (Guillebaud, 2008). Bien évidemment, le mouvement des populations et des collectivités est un effet constant dans l'histoire de l'Occident, mais il présente aujourd'hui des caractéristiques particulières : l'émergence de la pluralité dans les sociétés et de la diversité des individus qui la composent, loin de s'estomper avec la modernité, pose des défis nouveaux en termes de vivre ensemble et invite à revoir les fondements des relations entre identités, altérités et pluralités (Rossi, 2003).

La seconde raison repose sur le constat que, dans notre monde contemporain, s'il est " une norme, une valeur et une aspiration universelle aujourd'hui c'est bien celle de la santé qui, en quelques décennies, a touché les populations de tous les continents, les riches comme les moins aisés » (Hours, 2004). Selon l'Organisation mondiale de la santé (OMS), le concept de santé désigne un état de bien-être physique, psychologique et social et représente donc davantage que l'absence de troubles, de maladies ou encore de handicaps. Bien entendu, cet état constitue un idéal à atteindre et intègre le concept de qualité de vie. Au-delà de l'universalité évoquée, cette vision cache en fait une complexité souvent ignorée. Dans son acception moderne, la santé renvoie aux systèmes de prévention, de traitement et de gestion du malheur, compris simultanément comme des systèmes culturels et des systèmes sociaux, qui donnent au corps, à la santé et à la maladie leur sens, leurs formes et leurs évolutions (Saillant et Genest, 2006). C'est donc à la santé au sens large - non seulement individuelle, mais aussi publique et collective que l'on se référera.

Les déterminants de la santé d'une personne, à un moment donné, sont aussi bien endogènes (psychologiques et biologiques) qu'exogènes (facteurs sociaux, économiques, culturels, politiques). Concernant ces derniers, des pressions socioéconomiques persistantes sont des facteurs de risque reconnus pour la santé mentale des individus et des collectivités. Les données factuelles qui l'attestent le mieux sont les indicateurs de pauvreté, et en particulier, les faibles niveaux d'éducation (Marmot, 2006). Par conséquent, la promotion de la santé passe non seulement par l'accès à des traitements individuels, mais aussi et d'abord par des actions d'ordre politique, économique et social tendant à promouvoir des conditions de vie et un environnement favorisant un mode de vie

\footnotetext{
${ }^{1}$ À la suite d'un colloque interfacultaire du même titre qui s'est tenu à l'Université de Genève (UNIGE) et à l'Université de Lausanne (UNIL), respectivement les 13 et 14 novembre 2013.
} 
sain et diminuant les facteurs de risque. Ainsi, un contexte garantissant le respect et la protection des droits civils, politiques, socioéconomiques et culturels fondamentaux est indissociable de mesures politiques visant à favoriser la santé. Il s'agit notamment d'intégrer la promotion de la santé aux politiques et aux programmes des secteurs public et non gouvernemental, mais aussi au niveau des pratiques professionnelles. La promotion de la santé repose pour une large part sur des stratégies intersectorielles, c'est-à-dire mettant en lien le secteur de la santé avec un autre secteur, afin d'intervenir indirectement sur un problème de santé. Dans cette approche transversale et globale, il convient d'associer au secteur de la santé les secteurs suivants : éducation, emploi, justice, transports, environnement, logement et protection sociale (McMichael et Beaglehole, 2000). Par exemple, améliorer le logement, l'urbanisme, l'alimentation, l'accès à l'eau potable, l'agriculture ou l'éducation peut avoir un impact indirect sur l'incidence des pathologies (OMS, 1997).

Dans son acception publique, la santé requiert donc une gouvernance collective qui distingue ce qui relève de la maladie individuelle de ce qui favorise la gestion sanitaire des collectivités et des populations. Elle témoigne de l'importance de l'économie et des responsabilités du politique - au niveau global, continental, régional, national dans l'élaboration de stratégies, dans l'organisation des institutions de soins et dans la formation des professionnels. Dès lors, elle se définit comme une construction sociale et politique dont les limites sont constamment redéfinies. Dans cette perspective constructiviste, la santé devient un objet socialement élaboré, historiquement construit, dont les limites mouvantes sont elles-mêmes régulièrement débattues (Fassin, 2000). La notion de santé est donc un outil heuristique, un levier de compréhension des enjeux politiques, économiques et culturels des sociétés, dépassant les limites de la maladie et des soins.

La notion de santé mentale se renouvelle également. Longtemps marquée par l'idée d'un fonctionnement optimal du psychisme, par une vision qui opposait normal et pathologique et par une institutionnalisation psychiatrique (Otero, 2003), elle se complexifie et ne peut désormais plus faire l'économie de considérations d'ordre bio-psychosocial et systémique. Les modèles actuels sont multifactoriels et mettent en jeu corps, patrimoine génétique, psychisme, émotions, environnement social, culturel, économique et écologique ou encore habitudes, comportements et qualité de vie, accès aux soins et qualité des soins. Dans la perspective décrite plus haut, la santé mentale est un état de bien-être dans lequel une personne peut se réaliser, surmonter les tensions normales de la vie, accomplir un travail productif et contribuer à la vie de sa collectivité (OMS, 2014). En ce sens positif, la santé mentale est le fondement à la fois du bien-être individuel et du bon fonctionnement social. Cela sous-entend que santé et social sont indissociables et qu'une qualité de vie minimale est indispensable au bon fonctionnement psychique. D'ailleurs, un numéro spécial de l'International Review of Psychiatry paru fin 2014, intitulé Globalization, culture and mental health, souligne dans ses différentes contributions à quel point les problèmes de santé mentale sont associés aux éléments suivants : changement social rapide, conditions de travail éprouvantes, discriminations, exclusion sociale, mode de vie malsain, environnements violents, mauvaise santé physique et violations des droits de l'Homme (Tribe, 2014). La santé mentale se situe ainsi à la croisée de différents champs disciplinaires psychiatrie, psychologie, sociologie, économie, travail social - et les stratégies à mettre en œuvre pour l'analyser sont largement intersectionnelles.

La portée majeure de ce renouveau conceptuel concerne cependant la relation de soins, dans laquelle personnes concernées et problématiques sont de plus en plus diversifiées et plurielles, étant donné les profondes mutations culturelles et sociales résultant de la mobilité. Repenser les relations de soins dans un contexte de transformations et de diversification sociales est la posture selon laquelle se déploie ce numéro interdisciplinaire.

Santé mentale et sociétés plurielles deviennent ainsi des notions concordantes et convergentes : elles partagent en toile de fond une même "modernité métisse " (Guillebaud, 2008), qui refuse l'opposition des dualismes - nous et les autres - et trouve ses marques en élaborant les tensions ou les contradictions que ces échanges suscitent. Cette modernité rencontre aujourd'hui une pertinence certaine, sans doute parce qu'elle signale « la confrontation à des réalités que nous ne faisons pour l'instant que pressentir [...] ॥ (Bonniol, 2011, p. 14). II s'agit donc de renforcer la lecture qui affranchit la différence culturelle des représentations qui lui sont généralement associées, notamment en termes d'essentialisation des identités et des différences, pour en saisir les dynamiques et en percevoir les potentialités. II nous semble important de reprendre le postulat de Leanza (1999) selon lequel la notion de culture doit se penser de nos jours en fonction de trois caractéristiques : I’hétérogénéité, la perméabilité et le métissage. 
Hétérogénéité : Contrairement à une idée reçue, une culture ou une société est tout sauf homogène. Au sein de chaque groupe culturel, il existe une variété de classes d'âge, de classes sociales, de classes professionnelles, de groupes politiques ou religieux, de situations familiales, etc., et tous ces groupes sociaux participent à la même culture globale (Cuche, 1996, p. 50), qui est la résultante des contacts et interactions entre ces différents groupes sociaux. Tout membre d'une culture a des liens avec plusieurs sous-groupes socioculturels.

Perméabilité : le propre d'une culture est de se reproduire pour assurer la survie du groupe culturel et de définir des limites pour se différencier des autres groupes culturels. Aucun système culturel ne vit pour autant en vase clos : il doit se repenser, se renouveler, se régénérer en laissant la possibilité à des individus - y compris des nonmembres - d'y accéder et de l'influencer de l'intérieur.

Métissage: dans une optique dynamique, interactionniste et subjectiviste de la culture, celle-ci n'est pas une donnée figée sur laquelle les individus n'ont aucun pouvoir. Au contraire, l'accent est mis sur les échanges entre individus et entre groupes culturels qui s'approprient la culture, empruntent, rejettent et interprètent tel ou tel aspect à leur manière. Ainsi, la culture évolue et se métisse en permanence, tout en gardant, aux yeux de ses membres, le sens d'une identité.

Hétérogénéité, perméabilité et métissage caractérisent donc à nos yeux la "culture contemporaine " et ses déclinaisons plurielles de la diversité, de ses enjeux et de ses usages. En effet, la conception d'une culture ou d'une identité culturelle statique, sous-jacente au discours sur les différences culturelles, est vivement critiquée par les tenants de l'approche transnationale et postcoloniale (Bhatia, 2007; Glick-Schiller, Basch et Szanton-Blanc, 1995; Hermans et Kempen, 1998). Ces auteurs dénoncent la fréquente assimilation de la culture à une nation, et une vision de l'identité hors du temps et de l'espace. À l'inverse, ils soulignent sa nature dynamique, en perpétuel remaniement, et inscrivent les questions identitaires dans un monde aux interconnexions multiples et mouvantes, caractérisées de plus en plus par les interactions entre niveau local et global. Les concepts de self dialogique (Bhatia et Ram, 2001; Hermans, 2001) et de métissage (Chanson, 2011); Laplantine et Nouss, 2007) qui en résultent mettent l'accent sur le fait que la bi- ou multi-culturalité impliquent l'idée de l'acceptation de l'autre en soi, ou l'internalisation de la voix des autres, tout comme elle présuppose, en situation transculturelle, l'idée de transformation de pratiques et de significations culturelles, de métamorphose, de tensions voire de conflits sociocognitifs à élaborer et à dépasser (Skandrani, 2013).

Toutefois, nous constatons que la différence culturelle est encore trop fréquemment invoquée par les intervenants médico-psycho-sociaux ou par ceux travaillant dans les milieux de l'éducation comme un facteur explicatif de nos impasses et de nos difficultés. La mise en avant de la différence culturelle génère à la fois un sentiment d'impuissance et de frustration et peut provoquer des réactions défensives chez les professionnels (CohenEmerique et Hohl, 2004). Les conflits et tensions découlant des contacts interculturels font, au mieux, appel à des pratiques de médiation, qui sont tantôt conçues comme un arbitrage mou, tantôt au contraire comme la nouvelle utopie d'une société libérée de ses conflits (Younès, 2002).

\section{Un nécessaire changement de paradigme : de la différence culturelle à la diversité. L'exemple de la Suisse}

Les enjeux interculturels et les débats politiques et sociétaux engendrés par la présence de migrants dans nos sociétés occidentales ont évolué dans le temps. Grâce à une approche sociohistorique, Sane (2013) montre comment la notion de différence culturelle " problématique » et sa gestion est un construit qui reflète le regard que nous portons sur les étrangers et non une conséquence naturelle de la diversité ou de l'altérité culturelle. Retraçant l'histoire de l'immigration au $20^{\mathrm{e}}$ siècle en Suisse, Piguet (2004) montre comment, à partir des années 1980, le rapport entre les émigrés et la société d'accueil se modifie avec l'immigration de longue durée, alors qu'auparavant ceux-ci étaient « invisibles » et non « problématiques » car ils disposaient d'un statut temporaire.

L'arrivée des femmes et des enfants donne lieu à l'émergence d'une nouvelle problématique, celle de la distance entre les caractéristiques sociales et culturelles des familles migrantes et les valeurs dominantes de la société de résidence. L'accroissement de leur visibilité en tant qu'usagers des institutions sociales (écoles, crèches, 
maternités, services de la jeunesse, hôpitaux, filières de formation, etc.) va engendrer des interrogations quant à leur intégration et à la compatibilité de leurs pratiques culturelles avec les idéaux de laïcité ou d'égalité et de liberté de la société de résidence, notamment en matière de structure familiale, de rapports entre les sexes et de rôles parentaux. Dès lors vont émerger des tensions et des problèmes liés à la différence culturelle en lien avec la reproduction par les migrants de certaines pratiques comme la polygamie, l'excision et, ultérieurement, le mariage forcé, le port du voile ou les châtiments corporels des enfants. L'attitude jusque-là tolérante envers ces pratiques au nom du relativisme culturel va se transformer en un rejet, un " choc » envers ce qui apparaît dès lors comme des pratiques déviantes. Dans un contexte d'essor de la victimisation, des associations féministes dénoncent ce qui est thématisé comme des violences intrafamiliales insupportables, inacceptables, et demandent que soient respectés les droits des plus faibles, à savoir les femmes et les enfants. C'est par le prisme de la violence exercée sur les femmes que seront dénoncées et portées sur le devant de la scène publique des pratiques culturelles jugées dégradantes à leur égard. Ces thèmes seront repris par des discours politiques visant des enjeux, non plus d'intégration et du vivre ensemble, mais de fermeture des frontières.

Depuis le début des années 1990, à l'instar d'autres pays occidentaux, la Suisse est en outre concernée par l'arrivée d'un nombre croissant de requérants d'asile et de travailleurs européens, qualifiés comme non qualifiés. En effet, après dix ans de négociations, la Suisse a signé avec l'Union européenne des accords bilatéraux l'associant à l'espace Schengen de libre circulation et permettant aux ressortissants des pays européens de s'établir sur son territoire. De fait, à l'exception des droits politiques, les immigrants européens ont désormais les mêmes droits que les nationaux et vice versa. Nous assistons donc à la désagrégation du lien entre secteur économique et immigration d'une part, et à la diversification des motifs d'immigration d'autre part. Dorénavant, la participation de la Suisse au système international la soumet aux exigences de l'unification européenne et du droit humanitaire et la force à s'aligner sur la politique de ses voisins. Les ralentissements conjoncturels et les récessions économiques ne peuvent plus être amortis par la fermeture des frontières aux travailleurs immigrés européens. Toutefois, cette tentation demeure présente, comme le montrent les résultats d'une récente votation populaire au niveau national intitulée «Contre l'immigration de masse ».

En résumé, avec le développement de l'immigration familiale et d'asile, les migrants sont devenus des usagers des institutions suisses. Leur "culture ", c'est-à-dire leurs us et coutumes, qui avait fait jusqu'alors l'objet de peu d'intérêt de la part des autochtones, devient dès lors «visible » par les professionnels de ces institutions, qui se retrouvent en première ligne, souvent sans formation pour penser et agir en situation d'interculturalité. Certaines pratiques " problématiques " génèrent des tensions autour de la question de la compatibilité de ces pratiques avec les cadres de référence, valeurs et idéaux des sociétés d'accueil, repris sous le terme d'intégration.

Qu'en est-il des effets de la stabilisation des permis de séjour sur l'intégration des migrants? Alors que la pierre d'achoppement du débat se situe autour des questions de l'accueil et de l'intégration, les études montrent que les immigrants, et surtout leurs descendants, ont remarquablement trouvé une place au sein de la société helvétique (Bolzman, Fibbi et Vial, 2003). On observe une convergence des niveaux de formation par rapport aux autochtones ainsi qu'une bonne distribution dans le monde du travail et une maîtrise de la langue. Il existe toutefois des ombres au tableau : les statistiques font état d'une surreprésentation des étrangers parmi les auteurs d'infractions et les personnes condamnées pénalement (Kuhn, 2012) et, de même, une surreprésentation des étrangers parmi les personnes présentant des problèmes de santé (Enquête suisse sur la santé) et certains troubles psychiques (Gabadinho, Wanner et Dahinden, 2007). On constate également une surreprésentation d'élèves d'origine étrangère parmi les enfants qui redoublent ou qui sont orientés vers des filières d'éducation spécialisée (Coradi Vellacott et Wolter, 2005; PISA, 2013) et des femmes migrantes parmi les personnes en recherche d'emploi (OCDE, 2012). Ces phénomènes, constatés de manière récurrente dans différents pays occidentaux, ont des origines complexes et multifactorielles qui laissent une large place aux inégalités sociales. La plupart des auteurs soulignent que l'intégration sociale et économique et la réussite scolaire sont rendues plus ardues par la discrimination - en particulier en termes de formation et d'emploi - et l'absence d'égalité des chances, obstacles qui peuvent susciter, chez certains, des mécanismes de marginalisation ou d'auto-exclusion (Furtos, 2008).

En ce début de $21^{\mathrm{e}}$ siècle, dans un contexte de mondialisation économique et de climat de durcissement des politiques migratoires et de fermeture des frontières, les enjeux autour de la diversité, en particulier culturelle, se 
sont complexifiés et amplifiés. Compte tenu des mutations contemporaines, bien que le « fait migratoire » ne soit pas nouveau, le discours politique sur ce phénomène a évolué, tout comme la valence qui y est associée. La notion de diversité succède à celle de différence culturelle. Contrairement à cette dernière, qui véhicule souvent l'idée d'un fossé voire d'une menace d'affaiblissement de l'identité nationale supposée homogène, la notion de diversité est considérée dans le discours institutionnel et politique progressiste comme un atout, découlant à la fois de la pluralité interne de nos sociétés modernes et de la reconnaissance du caractère pluriel du Sujet social. Cette reconnaissance et cette valorisation de la diversité sont en même temps au fondement d'un discours politique visant à renforcer la cohésion sociale par l'idéologie du vivre ensemble et de l'égalité des chances, qui sont censés faire reculer les inégalités et les discriminations. Dans cette optique, la "gestion de la diversité » renvoie essentiellement au domaine du politique, autour des concepts d'intégration et de respect du droit des groupes minoritaires (femmes, homosexuels, handicapés, étrangers) dans leur rapport au groupe dominant représenté par les instances étatiques. Mais cette question se pose aussi, nous l'avons vu, dans certaines situations concrètes, parfois difficiles, que les acteurs de terrain peuvent rencontrer. II nous semble, comme le soulignent Licata et Heine (2012), que l'attitude adéquate lors d'une intervention interculturelle consiste moins à critiquer les positions de l'Autre, ou à renoncer à ses propres valeurs, qu'à trouver le moyen de les traduire en actes qui tiennent compte de la différence culturelle entre soi et l'Autre. Le recours à un interprète peut constituer l'un de ces actes, permettant à la rencontre interculturelle de devenir un espace où le " fossé culturel » peut être approché, visité et parcouru par le discours et la pensée des uns et des autres, faisant de l'expérience de la différence culturelle et de l’altérité des contenus élaborables, sans risque de s'y perdre (Goguikian Ratcliff, 2010).

\section{Différence culturelle, santé mentale et diversité : quels paradigmes de recherche?}

$\mathrm{Au}$ cours des trois dernières décennies, les sciences sociales, humaines et médicales se sont nourries d'une distinction, mise en avant par des anthropologues de la santé, entre trois mots anglais qui désignent la maladie : disease, la pathologie définie par la médecine, illness, l'expérience subjective du patient, et sickness, le phénomène social qui définit le rôle du malade et les attentes de la société (Kleinman, Eisenberg et Good, 2006; Zempleni, 1985). L'analyse de ces trois dimensions fondamentales de la maladie est pertinente pour mettre en relief une approche à la fois scientifique et médicale rendant compte d'un problème de santé biologique ou psychique et constructiviste, témoignant du rôle des agents sociaux à faire exister un problème de santé et à y répondre. Ainsi comprise, la santé publique s'inscrit dans un double franchissement, celui qui distingue le social du médical et celui qui sépare le domaine du privé de la sphère publique (Dozon et Fassin, 2001). Si la maladie (illness) est individuelle, nous comprenons donc bien que la santé, elle, est collective : le statut, la place et l'expérience de l'humain face à la santé et à la maladie, les modes de réponses sociales et institutionnelles se reconfigurent dans une logique de l'offre et de la demande, fortement influencée par les transformations sociales et culturelles contemporaines.

La santé mentale, prise comme objet d'étude, permet d'accéder aux pratiques et aux discours des agents sociaux, incluant les interrelations entre usagers et professionnels et les politiques à l'œuvre dans les structures hospitalières. Son étude requiert donc une approche multifactorielle, voire pluridisciplinaire. Face à la complexité de la problématique, les chercheurs tentent d'élaborer, de décrire, voire de mesurer les processus qui entourent et construisent ces réalités à la fois interpersonnelles et sociales. Il est frappant de constater la variété sémantique, épistémologique et méthodologique qui se dégage des travaux effectués dans le domaine des approches dites interculturelles. Pour Vatz Laaroussi, Riard, Gélinas et Jovelin (2013), la question se pose de savoir si les notions de diversité culturelle ou d'interculturalisme sont des concepts-valises qui se diluent dans la multitude de facettes ou de variables confondantes qui les composent ou s'ils contribuent à de nouvelles configurations théoriques et méthodologiques en émergence. Ces auteurs proposent de ramener les travaux dans le domaine de la recherche interculturelle, en dépit de leur variété, à trois grands paradigmes, qui à notre avis vont du plus " neutre » au plus " engagé ». Le premier, plutôt positiviste et fonctionnaliste, se réfère à la diversité comme à une donnée factuelle qu'il faut gérer et canaliser, et avec laquelle il faut composer. C'est le cas des études faites de manière descriptive ou comparative sur l'état de santé de différentes populations consultantes en institution. Le deuxième, interactionniste, constructiviste et subjectiviste, met en avant les processus de reconnaissance, de participation et de construction réciproques des acteurs en présence et la mise en place d'un vivre ensemble harmonieux. C'est le cas, par exemple, des travaux sur l'acculturation et les stratégies identitaires, ou sur le travail psychothérapeutique avec des interprètes. Le troisième, sociocritique, aborde la diversité par les rapports sociaux de domination et

Alterstice - Revue Internationale de la Recherche Interculturelle, vol. $4, n^{\circ} 2$ 
associe les relations interculturelles aux mécanismes d'inégalité sociale et aux dynamiques de racisme et de discrimination. Nous retrouvons ici le cadre de la psychologie sociale ou encore les travaux sur l'intersectionnalité avec une approche genrée. Chacun de ces paradigmes a une légitimité, une validité intrinsèque et une valeur heuristique dans la description et la compréhension des phénomènes liés à la diversité et à l'interculturalité, pour autant qu'il ne s'érige pas comme vision unique.

Au sein de la relation soignante, la question du rapport à l'Autre se pose en termes de pouvoir et de reconnaissance, de prescriptions et de négociations. Face au patient, le professionnel incarne l'institution et, audelà, les valeurs dominantes de la société. Comment reconnaît-il la diversité des usagers et interagit-il en fonction d'elle? Dans un contexte de mondialisation, il nous semble important d'accorder une attention particulière aux processus de transition, de transmission, de transformation, de créativité, d'emprunts, de co-construction, de métissage. Cela revient à étudier la construction de l'interculturalité en situation d'interaction sociale, c'est-à-dire les rapports entre individus, ceux entre groupes de population dominants et minoritaires ou encore ceux entre usagers et professionnels lorsque ceux-ci ne se réfèrent pas aux mêmes normes et valeurs socioculturelles.

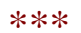

Nous proposons dans ce numéro un regard pluriel sur la diversité, que ce soit au niveau des concepts, des modèles, des politiques mises en place ou des pratiques observées dans nos sociétés plurielles, et ce, avec des auteurs appartenant à des disciplines différentes. Cette ouverture repose sur un double questionnement, éthique d'une part - face à l'émergence des sociétés plurielles, comment conceptualiser, globaliser et problématiser le concept de diversité dans le domaine de la santé mentale? - et scientifique d'autre part - cette notion spécifique peut-elle aujourd'hui être déterminante pour l'élaboration d'un savoir critique et autocritique en santé mentale?

Philippe Chanson, anthropologue, décline les généalogies théoriques et les métaphores successives du concept de métissage en sciences humaines: il nous montre la variabilité sémantique d'une notion et, par là même, le sens qu'elle assume en fonction des filières scientifiques et des contextes sociaux pluriels. Procédant par analogies, sa perspective permet de figurer et de complexifier la notion, et de poser les jalons d'une pensée contemporaine du métissage.

Marika Moisseeff, anthropologue et psychiatre, adopte une perspective sociohistorique pour souligner la nécessité de se détacher d'une posture qui inscrit la différence culturelle dans une hiérarchisation des races sous-tendue par une pensée évolutionniste. Par l'analyse des dérives d'une telle tentative, elle illustre a contrario la nécessité de penser la diversité à travers le prisme de l'égalité, notion dont elle est indissociable, et conclut par quelques pistes concernant la relation de soin.

Ilario Rossi, anthropologue, nous invite à une réflexion contextualisée de la relation de soin en santé mentale. II propose une lecture systémique des interfaces entre économie, technologie, science et clinique; il souligne ainsi le risque du primat d’uniformité, de standardisation et d'efficience sur la nécessité de penser la diversité comme subjectivité et corporéité.

Lise Demailly, sociologue, axe son propos sur la précarisation grandissante de nos sociétés occidentales contemporaines et sur l'enchevêtrement entre immigration et exclusion sociale et économique. Elle déconstruit et dénonce les logiques sous-jacentes à l'uniformisation des politiques de santé et celles qui régissent la gestion du relationnel.

Betty Goguikian Ratcliff, Claudio Bolzman et Théogène Gakuba, psychologues (1 et 3) et sociologue (2), se penchent sur la situation des femmes dans la migration internationale. Ils montrent que celles-ci, lors de leurs tentatives d'insertion professionnelle dans le pays d'accueil, subissent des discriminations multiples liées à la fois au genre et au statut d'immigrée, qui débouchent parfois sur un phénomène de déqualification touchant plus particulièrement les migrantes originaires de pays extra-européens. Ils soulignent les conséquences psychologiques de ces difficultés d'insertion. 
Javier Bartolomei, Rachel Baeryswil-Cottin et Natacha Premand, psychiatres (1 et 3) et psychologue (2), montrent la nécessité de la part des professionnels et des institutions de la santé mentale d'adapter les connaissances psychopathologiques et scientifiques à une nouvelle réalité, de manière à développer des modèles de soin aptes à considérer simultanément les tableaux cliniques, les souffrances psychiques, les trajectoires de migration et la variabilité culturelle.

\section{Références}

Abélès, M. (2008). Anthropologie de la globalisation. Paris : Payot.

Bhatia, S. (2007). Rethinking Culture and Identity in Psychology: Towards a Transnational Cultural Psychology. Journal of Theoretical and Philosophical Psychology, 27-28(2-1), 301-321.

Bhatia, S. et Ram, A. (2001). Rethinking "acculturation" in relation to diasporic cultures and postcolonial identities. Human Development, 44, 1-17.

Bolzman, C., Fibbi, R. et Vial, M. (2003). Que sont-ils devenus ? Le processus d'insertion des adultes issus de la migration. In H.R. Wicker, R. Fibbi et W. Haug (dir.), Les migrations et la Suisse (p. 79-100). Zurich : Seismo.

Bonniol, J.-L. (2011). Préface. Dans P. Chanson, Variations métisses. Dix métaphores pour penser le métissage (p. 13-21). Louvain-la-Neuve : Bruylant-Academia.

Chanson, P. (2011). Variations métisses. Dix métaphores pour penser le métissage. Louvain-la-Neuve : BruylantAcademia.

Cohen-Emerique, M. et Hohl, J. (2004). Les réactions défensives à la menace identitaire chez les professionnels en situations interculturelles. Les Cahiers internationaux de psychologie sociale, 61, 21-34.

Collier, S. et Ong, A. (dir.). (2005). Global assemblages : Technology, politics, and ethics as anthropological problems. Malden, MA : Blackwell.

Coradi Vellacott, M. et Wolter, S. (2005). L'égalité des chances dans le système éducatif suisse [en ligne]. Aarau : Centre suisse de coordination pour la recherche en éducation. http://www.skbfcsre.ch/fileadmin/files/pdf/publikationen/tb9_fr.pdf

Cuche, D. (1996). La notion de culture dans les sciences sociales. Paris : La Découverte.

Dozon, J.-P. et Fassin, D. (dir.). (2001). Critique de la santé publique. Une approche anthropologique. Paris : Balland.

Fassin, D. (2000). Entre politiques du vivant et politiques de la vie. Anthropologie et sociétés, 24(1), 95-116.

Furtos, J. (2008). Les cliniques de la précarité : contexte social, psychopathologie et dispositifs. Paris : Masson.

Gabadinho, A., Wanner, P. et Dahinden, J. (2007). La santé des populations migrantes en Suisse : une analyse des données du GMM : le rôle du profil sociéconomique, sociodémographique et migratoire sur l'état de santé, les comportements et le recours aux services de santé. Neuchâtel : Forum suisse des migrations.

Glick-Schiller, N., Basch, L. et Szanton-Blanc, C. (1995). From immigrant to transmigrant: Theorizing transnational migration. Anthropological Quarterly, 68, 48-63.

Goguikian Ratcliff, B. (2010). Différences culturelles fondamentales et exercice de la psychothérapie avec un interprète. In G. Thésée, N. Carignan et P. Carr (dir.), Les faces cachées de la recherche interculturelle. De la rencontre des porteurs de culture (p. 78-96). Paris : L'Harmattan.

Guillebaud, J.-C. (2008). Le commencement d’un monde. Vers une modernité métisse. Paris : Seuil.

Hermans, H. (2001). The dialogical self: toward a theory of personal and cultural positionning. Culture and Psychology, 16, 89-130.

Hermans, H. et Kempen, H. (1998). Moving cultures: The perilous problems of cultural dichotomies in a globalizing society. American Psychologist, 53, 1111-1120.

Hours, B. (2004). Trois objets-étapes de la globalisation de la norme de santé. Autrepart (1), 135-144.

Alterstice - Revue Internationale de la Recherche Interculturelle, vol. 4, $n^{\circ} 2$ 
Hugo, G. (2005). Migrants in society: diversity and cohesion. Genève : Commission mondiale sur les migrations internationales.

Kleinman, A., Eisenberg, L. et Good, B. (2006). Culture, Illness and Care: clinical lessons from anthropologic and cross-cultural research. Focus, 4, 140-149.

Kuhn, A. (2012). Comment s'explique la surreprésentation des étrangers dans la criminalité? [en ligne]. Genève : Vivre ensemble, Service d'information et de documentation sur le droit d'asile. http://www.asile.ch/vivreensemble/2012/10/06

Laïdi, Z. (2004). La grande perturbation. Paris : Flammarion.

Laplantine, F. et Nouss, A. (1997). Le métissage. Paris : Flammarion.

Leanza, Y. (1999). Situations interculturelles dans le monde de la santé : quelles approches pour quel rapport à l'Autre ? Poster présenté au VII' congrès de l'Association pour la Recherche InterCulturelle, Paris.

Licata, L. et Heine, A. (2012). Introduction à la psychologie interculturelle. Bruxelles : De Boeck.

Maier, T., Schmidt, M. et Mueller, J. (2010). Mental health and healthcare utilization in adult asylum seekers. Swiss Medical Weekly, 140, w13110.

Marmot, M. (2006). Health in an unequal world. The Lancet, 368, 2081-2094.

McMichael, A. et Beaglehole, R. (2000) The changing global context of public health. The Lancet, 356, 495-499.

Organisation de coopération et de développement économiques (OCDE) (2012). Perspectives des migrations internationales. Paris : OCDE. doi:10.1787/migr_outlook-2012-fr

Organisation mondiale de la santé (OMS). (1997). Intersectoral action for health. Conférence Halifax. OMS.

Organisation mondiale de la santé (OMS). (2014). La santé mentale : renforcer notre action [en ligne]. OMS (aidemémoire $n^{\circ}$ 220). http://www.who.int/mediacentre/factsheets/fs220/fr/

Otero, M. (2003). Les règles de l'individualité contemporaines. Santé mentale et société. Québec: Presses de I’Université Laval.

Piguet, E. (2004). L'immigration en Suisse. Cinquante ans d'entrouverture. Lausanne : Presses polytechniques et universitaires romandes.

PISA (2013). Premiers résultats tirés de PISA 2012 [en ligne]. Berne : SEFRI/CDIP et Neuchâtel : Consortium PISA.ch. http ://www.edudoc.ch/static/web/ak-tuell/medienmitt/ergebnisse_pisa2012_f.pdf

Rossi, I. (2003). Mondialisation et sociétés plurielles ou comment penser la relation entre santé et migration. Médecine et Hygiène, 2455, 2039-2044.

Saillant, F. et Genest S. (dir.) (2006). Medical Anthropology. Regional perspectives and shared Concerns. Malden, Oxford : Blackwell Publishing.

Sane, A. (2013). De la diversité aux problèmes interculturels : I'évolution des enjeux politiques et sociétaux de I’immigration africaine en France. Dans M. Vatz Laaroussi, E.-H. Riard, C. Gélinas et E. Jovelin (dir.), Les défis de la diversité : enjeux épistémologiques, méthodologiques et pratiques (p. 91-102). Paris : L'Harmattan.

Skandrani, S. (2013). L'identité en question. Critiques et nouvelles approches de l'identité en contexte transnational. Dans M. Vatz Laaroussi, E.-H. Riard, C. Gélinas et E. Jovelin (dir.), Les défis de la diversité : enjeux épistémologiques, méthodologiques et pratiques (p. 65-80). Paris : L’Harmattan.

Tribe, R. (2014). Globalization, culture and mental health. International Review of psychiatry, 26(5), 535-537.

Vatz Laaroussi, M., Riard, E-H., Gélinas, C. et Jovelin, E. (2013). Introduction. Dans M. Vatz Laaroussi, E.-H. Riard, C. Gélinas et E. Jovelin (dir.), Les défis de la diversité : enjeux épistémologiques, méthodologiques et pratiques (p. 11-19). Paris : L'Harmattan. 
Younès, C. (2002). Introduction. Dans C. Younès et E. Le Roy (dir.), Médiation et diversité culturelle, pour quelle société ? (p. 7-15). Paris : Karthala.

Zempleni, A. (1985). La "maladie" et ses "causes". L'ethnographie, 81(2-3), 13-44. 